\title{
Tendências e Variabilidades nas Séries Históricas de Precipitação Mensal e Anual no Bioma Cerrado no Período 1977-2010
}

\author{
Juliana de Oliveira Campos $^{1}$ (D) , Henrique Marinho Leite Chaves ${ }^{1}$ \\ ${ }^{1}$ Departamento de Engenharia Florestal, Faculdade de Tecnologia, Universidade Federal de \\ Brasília, Campus Darcy Ribeiro, Brasília, DF, Brasil.
}

Recebido em: 29 de Agosto de 2018 - Aceito em: 25 de Outubro de 2019

\begin{abstract}
Resumo
O objetivo deste trabalho foi analisar as séries históricas mensais e anuais de precipitação de longo termo do bioma Cerrado, buscando identificar tendências locais e regionais. Para tanto, foram realizadas diferentes análises de tendência de séries temporais de precipitação anual e mensal de 125 estações pluviométricas do Cerrado, no período entre 1977 e 2010. O teste de MK indicou que $71 \%$ dos postos analisados apresentaram tendência de queda na precipitação anual, dos quais 14\% apresentaram tendência de queda estatisticamente significativa, predominantemente na região centro oeste do bioma. Apenas $28 \%$ das estações apresentaram tendência de aumento, sendo que destas, $1 \%$ foi estatisticamente significativa. O teste de TS indicou que, em média, a precipitação total anual no Cerrado diminuiu $125 \mathrm{~mm}$ $(8,4 \%)$ no período estudado. Os meses de estação seca (junho, julho, agosto e setembro) apresentaram tendências de redução na precipitação em $76 \%$ das séries estudadas, sinalizando uma possível intensificação do período de estiagem na região. Os testes de tendência mostraram resultados consistentes entre si, sugerindo uma tendência geral de redução da precipitação no bioma.
\end{abstract}

Palavras-chave: Cerrado, tendência de precipitação, variabilidade climática.

\section{Trends and Variabilities in the Historical Series of Monthly and Annual Precipitation in Cerrado Biome in the Period 1977-2010}

\begin{abstract}
The objective of the present study was to analyze the annual and monthly precipitation historical series of the Cerrado biome (Brazil), in order to identify local and regional trends. Different trend detection methods were used for 125 rain gauge stations throughout the Cerrado during the period 1977-2010, including LOWESS (LW), Mann-Kendall (MK), Theil-Sen (TS), and Pettitt (PT). The MK test suggested negative trends for $71 \%$ of the stations, with $14 \%$ of them statistically significant, mainly in the southerly region. Positive trends were found in $28 \%$ of the stations, where $1 \%$ was significant. The TS test suggested that, in general, the annual total precipitation in Cerrado decreased by $8,4 \%$ during the studied period. The precipitation in the dry months (JJAS) decreased in $76 \%$ of the stations, indicating an intensification of the dry season in the region. The results were consistent, suggesting a general decreasing trend in the precipitation of the biome, during the studied period.
\end{abstract}

Keywords: Cerrado, precipitation trends, climate variability.

\section{Introdução}

As mudanças climáticas globais apresentam-se como um grande desafio para a humanidade. O $5^{\circ}$ Relatório (AR5) do Painel Intergovernamental sobre Mudanças Climáticas (IPCC) mostra que houve um aumento de
$0,85{ }^{\circ} \mathrm{C}$ na temperatura média global desde 1880 (IPCC, 2014). Em média, $20-40 \%$ da população global vive em áreas que já tiveram um aquecimento de $1,5{ }^{\circ} \mathrm{C}$, considerando o período 2006-2015 (IPCC, 2018). Este aquecimento global, majoritariamente absorvido pelo oceano, 
tem ocasionado uma série de impactos climáticos, como derretimento de geleiras, aumento do nível do mar (aproximadamente $20 \mathrm{~cm}$ desde 1900), aumento das chuvas no Hemisfério Norte e nos países de latitudes médias (IPCC, 2014; Maleski e Martinez, 2017), além do aumento da frequência, intensidade e abrangência espacial de eventos climáticos extremos, como secas, chuvas intensas e enchentes (IPCC, 2012; IPCC, 2014; O'Gorman, 2015; Zandonadi et al. 2016; Alizadeh-Choobari e Najafi, 2018). Há também alertas relativos ao aumento global das ondas de calor, de eventos de chuvas intensas, do número de dias e noites quentes e secas (IPCC, 2012).

Os resultados das pesquisas sobre mudanças climáticas a partir de uma média global, todavia, não traduzem adequadamente as tendências climáticas em escala regional e local, pois o sistema climático em associação com os fatores físicos e ambientais de cada região tende a responder de forma diferente ao aquecimento (Hoffmann e Jackson, 2000). De uma forma geral, o aquecimento está associado com aumento de chuvas intensas devido ao maior vapor d'água e a capacidade do ar quente em armazená-lo (Alizadeh-Choobari e Najafi, 2018). Todavia, esses autores observaram que o aquecimento no Irã $\left(1,3{ }^{\circ} \mathrm{C}\right.$ no período entre 1951-2013) foi acompanhado de uma redução da frequência de chuvas intensas e aumento da frequência de chuvas fracas, em desacordo com o esperado.

Na América do Sul, observações indicam que nas últimas décadas houve um aumento da precipitação na região norte e redução na região sul (Lee et al. 2011). No Brasil, as pesquisas mostram que tem ocorrido nas últimas quatro décadas um aumento de chuvas na região sul e sudeste, enquanto que o centro-norte do país tem apresentado tendências de redução da precipitação (Ávila et al. 2016; Salviano et al. 2016; Zandonadi et al. 2016; Sá et al. 2018).

Nesse contexto, pesquisas recentes buscaram avaliar a ocorrência, causas e consequências de eventuais alterações no padrão de precipitação do Cerrado, bioma localizado no Brasil Central e de suma importância econômica e ambiental para o país. Lee et al. (2011) reportaram uma redução de aproximadamente $70 \mathrm{~mm}$ na precipitação anual na região do Cerrado nas últimas décadas, considerando a diferença entre as médias da precipitação total anual entre os períodos de 1993-2006 e 1979-1992, obtidas pelos dados do Projeto Climatológico da Precipitação Global (Adler et al. 2003). Debortoli et al. (2015), por sua vez, observaram tendências de redução significativa da precipitação no bioma no início e fim da estação chuvosa durante 1971-2006, indicando um prolongamento da estação seca no bioma. Similarmente, Penereiro et al. (2018) encontraram nos meses da estação seca (junho, julho e agosto - JJA) e no período de transição com a estação chuvosa (setembro, outubro e novembro - SON) tendências de redução na precipitação.
Por outro lado, no sul do estado do Mato Grosso (MT), porção que abrange o Cerrado, houve um aumento significativo das chuvas em alguns meses da estação seca (JJA) e chuvosa (janeiro, fevereiro e março - JFM) para o período de 1961-2011 (Salviano et al. 2016). Almeida (2012), analisando 33 séries históricas de precipitação no Centro-oeste do Brasil, região majoritariamente englobada pelo Cerrado, encontrou uma tendência generalizada de aumento dos índices de precipitação diária no período entre 1970-2011, embora poucos resultados tenham sido estatisticamente significativos.

Do exposto acima, observa-se que a maioria dos estudos indica a ausência de variações estatisticamente significativas na precipitação do bioma Cerrado, existindo resultados controversos quanto às tendências de aumento $\mathrm{e}$ reduções das chuvas identificadas nos períodos de seca, chuva e transição das estações. A divergência observada, no que se refere aos sinais das tendências, pode ter ocorrido em função do período estudado, da fonte de dados utilizada, de séries pluviométricas limitadas, ou dos métodos estatísticos aplicados para detectar as tendências nas séries históricas de precipitação. Entretanto, é de suma importância investigar alterações no regime de chuvas no Cerrado, dado a dimensão de seus potenciais impactos para o meio ambiente e as atividades humanas.

O Cerrado possui uma área total de 2 milhões de $\mathrm{km}^{2}$, compreendendo uma região crítica para o desenvolvimento socioeconômico do país e prioritária para conservação da biodiversidade e dos recursos hídricos. Sua rica vegetação caracterizada por um mosaico de fitofisionomias abriga 12.000 espécies de plantas e animais, das quais 4.800 são endêmicas (Strassburg et al. 2017). O Cerrado também tem papel primordial na segurança hídrica do país uma vez que contém nove das doze bacias hidrográficas brasileiras, das quais seis têm nascentes em seus limites (Lima, 2011; Brasil, 2011).

Do ponto de vista econômico, vale ressaltar que o bioma é responsável por $64 \%$ da criação de gado e $60 \%$ da produção de soja (Brasil, 2014; IBGE, 2016). Seu papel decisivo na agropecuária iniciou-se na década de $70 \mathrm{com}$ os incentivos governamentais para ocupação do Centrooeste do país, que resultou em mais de $50 \%$ de sua vegetação nativa substituída por usos antrópicos (Brasil, 2014; Arantes et al. 2016). Acredita-se que tais modificações no uso do solo do bioma poderão contribuir para mudanças significativas no clima do seu próprio território e de regiões vizinhas (Hoffmann e Jackson, 2000; Costa e Pires, 2010; Lee et al. 2011; Salazar et al. 2015; Arantes et al. 2016; Spera et al. 2016; Debortoli et al. 2016).

Tendo em vista o exposto, o objetivo desse estudo foi analisar as séries históricas mensais e anuais de precipitação de longo termo do bioma Cerrado, buscando identificar tendências locais e regionais que possam indicar se essa variabilidade já é uma realidade na região. 


\section{Material e Métodos}

\subsection{Obtenção e tratamento dos dados de precipitação}

Os dados mensais de precipitação do bioma Cerrado foram adquiridos através da ferramenta Hidroweb do Sistema Nacional de Informações da Agência Nacional das Águas-ANA. Uma checagem preliminar foi feita com a finalidade de eliminar estações que apresentavam uma quantidade significativa de valores inconsistentes, selecionar postos que apresentavam período de observação maior que 30 anos, e falhas que, consecutivamente, não ultrapassassem um ano (ANA, 2011).

Visando uma maior representação numérica e melhor distribuição espacial, bem como séries temporais com períodos idênticos e contínuos, optou-se por utilizar os 125 postos pluviométricos com dados de 34 anos contínuos (01/01/1977 a 31/12/2010) para as análises. Assim, o tamanho da série utilizada neste estudo satisfez o período mínimo de 30 anos recomendado pela Organização Meteorológica Mundial - WMO - para obtenção de normal climatológica (WMO, 1989). As 125 estações selecionadas para o estudo são apresentadas na Fig. 1.

Posteriormente, eventuais falhas de dados nos postos foram identificadas e preenchidas por dois métodos distintos: a) Método de Média Histórica Mensal - se a série pluviométrica apresentava falhas com até dois meses faltantes em um determinado ano, a precipitação desses meses foi substituída pela média histórica mensal respectiva (Río et al. 2011; Debortoli et al. 2015); b) Regressão Linear Simples - se a série pluviométrica apresentava falhas em mais de dois meses faltantes em um mesmo ano, esses meses foram preenchidos pelo valor estimado obtido a partir de um modelo de regressão com outra estação pluviométrica próxima (Tucci, 2012; ANA, 2011). Como prérequisito para aplicação deste último método, as duas estações deviam ter no mínimo 10 anos de dados em comum.

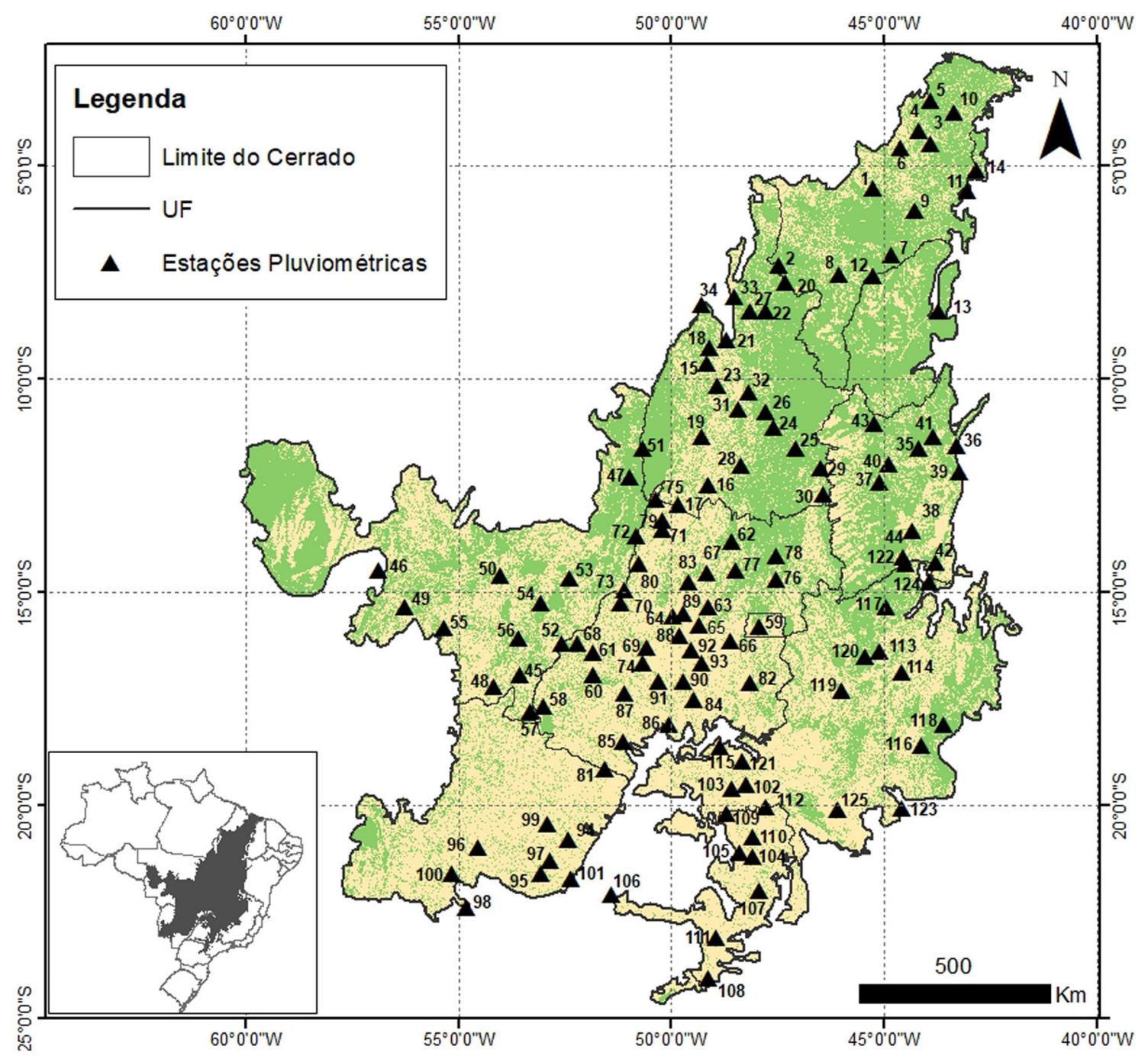

Figura 1 - Localização das 125 estações no bioma Cerrado utilizadas no estudo, com série de 34 anos (1977-2010). 
$\mathrm{O}$ resultado do preenchimento das séries foi considerado satisfatório quando o coeficiente de determinação foi superior a 0,7 (De Oliveira et al. 2010; Vilanova, 2014). Dentre as estações, nem todas tiveram $R^{2}$ superior a 0,7 e, por isso, antes de serem eliminadas do estudo, as estações foram submetidas à Análise de Consistência, a fim de verificar a confiabilidade dos dados. Para tal, foi utilizado o método de Dupla Massa (Sayemuzzaman e Jha, 2014), que indicou que todos os 125 postos apresentavam homogeneidade nos dados $\left(\mathrm{R}^{2}>0,99\right)$.

Após o tratamento dos dados, obteve-se um total de 1.638 séries temporais de precipitação, sendo: a) 125 séries referentes à precipitação anual de cada estação, resultado da soma dos totais mensais de cada um dos 34 anos; b) 1.500 séries mensais de precipitação (125 estações pluviométricas x 12 meses), para avaliar a dinâmica da precipitação nos meses chuvosos e secos; c) uma série de precipitação anual regional do bioma Cerrado, obtida a partir da média anual das 125 estações estudadas; e d) 12 séries mensais de precipitação regional (do bioma Cerrado), resultado da média das 125 estações para cada mês do ano.

\subsection{Análises das séries históricas de precipitação}

A partir dos dados de precipitação mensal e anual tratados, foi realizada uma análise exploratória usando o método LOWESS (Locally Weighted Scatter-plot Smoothing), o qual permite entender melhor a relação entre as variáveis da série histórica - Tempo $(T)$ versus Precipitação $(P)$, a partir de uma função de suavização (Kundzewicz e Robson, 2000). A curva LOWESS é obtida a partir da atribuição de um peso para um conjunto de vizinhos próximos a $x_{T}$ (quanto maior for a proximidade, maior o peso) e uma regressão baseada no peso para obter o valor de $y$ estimado. Dessa forma, quanto maior o número de vizinhos selecionados, maior a suavização (Shumway e Stoffer, 2011). No presente estudo, o uso do método LOWESS teve como objetivo avaliar o padrão e as tendências da precipitação ao longo da série histórica, selecionando um número de três vizinhos, para que as flutuações locais e eventuais mudanças abruptas fossem reduzidas (Taxak et al. 2014; Ahmad et al. 2015).

Para a detecção de aumento ou queda nas tendências das séries históricas de precipitação do Cerrado observadas, utilizou-se o teste não paramétrico de Mann-Kendall (MK), amplamente empregado por diversos estudos relacionados a essa temática (Mourato et al. 2010; Ahmad et al. 2015; Sanogo et al. 2015; Debortoli et al. 2015; Bisht et al. 2018). A hipótese nula $\left(\mathrm{H}_{0}\right)$ do teste é que não há tendência significativa na série, enquanto as hipóteses alternativas podem ser tendência de aumento ou diminuição significativa. Assim, considerando que uma série temporal de $(n)$ dados, onde cada valor da série $\left(x_{i}\right)$ é comparado com o valor subsequente $\left(x_{j}\right)$, a soma dos sinais (sgn) de todas as diferenças consideradas representa a variável estatística " $S$ " do teste de Mann-Kendall (Mann, 1945; Kendall, 1975), dada pelas Eqs. (1)-(2):

$$
\begin{aligned}
& S=\sum_{i=1}^{n-1} \sum_{j=i+1}^{n} \operatorname{sgn}\left(x_{j}-x_{i}\right) \\
& \operatorname{sgn}\left(x_{j}-x_{i}\right)= \begin{cases}+1 ; & \operatorname{se} x_{j}>x_{i} \\
0 ; & \operatorname{se} x_{j}=x_{i} \\
-1 ; & \operatorname{se} x_{j}<x_{i}\end{cases}
\end{aligned}
$$

A variância da série $(V(S))$ é calculada a partir da Eq. (3), dada pela relação:

$$
V(S)=\frac{n(n-1)(2 n+5)-\sum_{i=1}^{m} i(i-1)(2 i+5)}{18}
$$

onde $(m)$ é o número de grupos contendo valores iguais na série de dados, e ( $i$ ) representa a quantidade de repetições de certo grupo.

Mesmo para poucos dados na série $(n \geq 10)$, se pode assumir uma distribuição normal, na qual o índice de MK $\left(Z_{M K}\right)$ pode ser obtido com base nos sinais de $(S)$ (Eq. (4)).

$$
Z_{M K}= \begin{cases}\frac{S-1}{\sqrt{V(S)}} & \text { se } S>0 \\ 0 ; & \text { se } S=0 \\ \frac{S+1}{\sqrt{V(S)}} & \text { se } S<0\end{cases}
$$

Valores positivos de $\left(Z_{M K}\right)$ indicam uma tendência crescente na precipitação, ao passo que valores negativos de $\left(Z_{M K}\right)$ indicam uma tendência decrescente. A hipótese nula, de que não há tendência, é rejeitada quando $Z_{M K}>Z_{1}-\frac{\alpha}{2}$. Nesse estudo, o teste de MK foi usado para detectar tendências nas séries temporais de precipitação usando um intervalo de confiança de $95 \%$ de probabilidade $\propto=0,05$, o que faz necessário um valor crítico de $Z_{M K}>1,96$.

Com o objetivo de avaliar a magnitude da tendência das séries temporais de precipitação, foi aplicado o teste não paramétrico de Theil-Sen (TS) (Theil, 1950; Sen, 1968). O estimador de declividade de TS ( $\beta$ ) é calculado a partir da mediana de todas as declividades entre todos os pares de pontos na série, representado pela Eq. (5).

$$
\beta=\text { mediana }\left(\frac{x_{j}-x_{1}}{j-i}\right) \text { para todo } i<j<n
$$

onde $\left(x_{i}\right)$ e $\left(x_{\mathrm{j}}\right)$ representam os pontos na série temporal $(i)$ e $(j)$, respectivamente $(i<j)$. O valor de $\beta$ indica a declividade média anual da tendência da série, enquanto o sinal negativo indica um decréscimo na tendência e o positivo, um aumento. Assim, a redução da precipitação em milí- 
metros (mm) no período (1977-2010) é obtida multiplicando $\beta$ pelo número de anos totais (34 anos). De acordo com Taxak et al. (2014) e Bisht et al. (2018), a magnitude da mudança da precipitação em porcentagem $(\Delta x)$ ao longo do período analisado é adquirido pela Eq. (6).

$$
\Delta x=\frac{T x \beta}{E(x)}
$$

onde (T) é tamanho do período em anos e $(E)$ é a precipitação média anual da série do Cerrado no período em que se pretende analisar. Como sugerido por Sayemuzzaman e Jha (2014) e Sharma e Saha (2017), foi realizada uma análise espacial da magnitude da tendência da precipitação a partir da interpolação do coeficiente $\beta$ pelo método de Inverse Distance Weight (IDW), realizado no ambiente SIG (ArcGIS®, versão 10.2.2.).

Finalmente, foi aplicado o teste de Pettitt (Pettitt, 1979), para detectar o momento de ruptura das séries históricas, ou seja, o ponto de mudança de tendência na série, caso este existisse. $O$ teste consiste em dividir a série não estacionária original em duas séries estacionárias, que contêm distribuição e média diferentes (Kundzewicz e Robson, 2000). A hipótese nula $\left(\mathrm{H}_{0}\right)$ é de não ruptura na série, enquanto a hipótese alternativa $\left(\mathrm{H}_{1}\right)$, é que há ruptura. O método proposto por Pettitt (1979) se baseia numa reformulação do teste de Mann-Whitney, sendo a estatística do teste $\left(U_{t}\right)$ calculada com as Eqs. (7)-(8).

$$
U_{t}=\sum_{i=1}^{t} \sum_{j=1}^{t} \operatorname{sgn}\left(X_{i}-X_{j}\right) \text { para } t=2,3, \ldots n
$$

$$
\operatorname{sgn}=(x)\left\{\begin{array}{l}
1 \text { se } x>0 \\
0 \text { se } x=0 \\
-1 \text { se } x<0
\end{array}\right\}
$$

O ponto de ruptura mais significativo $\left(K_{t}\right)$ é identificado onde o valor de $\left|U_{t}\right|$ é máximo (Eq. (9)).

$$
K_{t}=\max \left|U_{t}\right|
$$

A probabilidade aproximada $(P)$ para o ano de ruptura na série de precipitação é dada pela Eq. (10).

$$
P=2 \exp ^{-6 K^{2} /\left(t^{3}+t^{2}\right)}
$$

O teste de Pettitt (1979) foi aplicado a todas as 126 séries temporais anuais (125 séries das estações individuais e uma série global média do Cerrado), ao nível de 5\% de significância. Todas as análises estatísticas foram realizadas no software R (versão 3.3.3), usando o pacote trend (Pohlert, 2018).
Os testes não paramétricos utilizados (Mann-Kendall, Theil-Sen e Pettitt) requerem que os dados de precipitação em dentro de uma mesma série temporal sejam não correlacionados, independentes no tempo (Gocic e Trajkovic, 2013). Assim, para avaliar a autocorrelação, utilizou-se a função "ACF" no pacote estatístico R (versão 3.3.3), a partir do cálculo da função de autocorrelação para o lag 1(r), considerando o nível de significância de $5 \%$. Esse cálculo foi aplicado para todas as séries pluviométricas, sendo que nenhuma é autocorrelacionada em relação ao tempo, permitindo-se, assim, que os testes não paramétricos fossem aplicados às mesmas.

\section{Resultados e Discussão}

\subsection{Precipitação média e distribuição da chuva no Cerrado}

A precipitação média anual em todo o bioma Cerrado, no período compreendido entre 1977 e 2010, foi de $1.486 \mathrm{~mm} \pm 146,8 \mathrm{~mm}$. A Fig. 2 indica que este bioma apresenta uma alta variabilidade espacial na precipitação anual, observando-se, entretanto, grupos de estações com médias semelhantes. A região central do Cerrado, principalmente o estado de Goiás, concentrou os valores médios de precipitação das séries $(1.442 \mathrm{~mm}$ a $1.780 \mathrm{~mm})$, os quais são característicos do bioma. Valores inferiores à média regional estão localizados nos estados do Piauí, Bahia e Minas Gerais (norte), que compreende a faixa de transição com o bioma Caatinga. Como esperado, valores superiores à média estão concentrados nas áreas de transição com a floresta amazônica. De maneira geral, a precipitação anual no Cerrado aumenta de leste para oeste. Um conjunto de estações com altos valores de precipitação (azul escuro) no oeste do Tocantins, incluindo médias pluviométricas de $2.400 \mathrm{~mm} / \mathrm{ano}$, indica que as maiores médias estão na região de transição entre o Cerrado e a Amazônia.

Em relação à precipitação média mensal, observouse uma alta amplitude no bioma Cerrado (dados não apresentados). No período chuvoso (dezembro, janeiro, fevereiro e março), a maior média mensal $(466 \mathrm{~mm})$ ocorreu no mês de dezembro (Posto 62 / Campinaçu - GO). Por outro lado, a média mais baixa deste mês foi $93 \mathrm{~mm}$ (Posto 10 / Chapadinha - MA). Durante a estação seca (JJA), o mês de julho teve a menor média pluviométrica mensal, próxima à zero em algumas estações: Posto 35 (Cotegipe BA), Posto 44 (Cocos - BA), Posto 39 (Ibotirama- BA), Posto 13 (Cristino Castro - PI) e Posto 124 (Manga - MG). Por outro lado, a estação 108 (Itaberá - SP) apresentou a maior média mensal para o mesmo mês, com $79 \mathrm{~mm}$. Os resultados também confirmam o clima de savana predominante no Cerrado, caracterizado por invernos secos e verões com as chuvas mais intensas (Da Silva et al., 2008). 


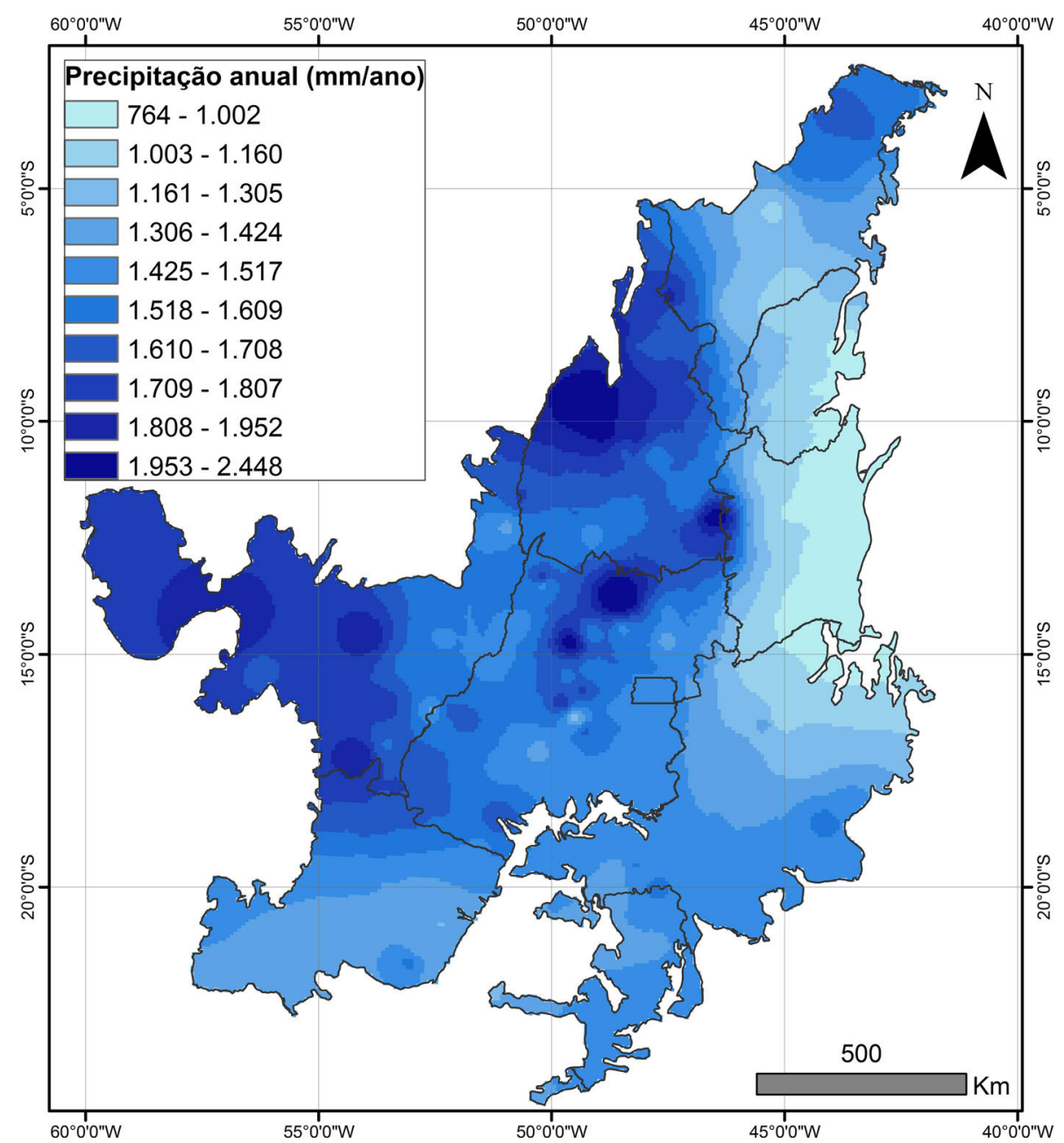

Figura 2 - Precipitação média anual $\left(\mathrm{mm} \cdot \mathrm{ano}^{-1}\right)$ no bioma Cerrado.

\subsection{Tendências nas séries históricas de precipitação}

A série temporal de precipitação anual do Cerrado, obtida a partir da média da precipitação anual das 125 estações, no período entre 1977 e 2010, é apresentada na Fig. 3. Inicialmente, observa-se uma variabilidade interanual inerente ao processo pluviométrico (Obregón e Marengo, 2011). Baixos valores de precipitação são observados nos anos de 1984, 1990 e 2007, os quais podem estar associados ao fenômeno El-Niño, enquanto que valores elevados, superiores à média pluviométrica de longo termo, foram observados nos anos de 1985 e 1989, possivelmente associados ao fenômeno La Niña (Oliveira et al. 2014; Debertoli et al. 2015). É possível notar ainda uma tendência de queda entre os anos de 1977 e 1998 na série, sendo que após esse período, ela se mantém relativamente estável até 2010.

$\mathrm{O}$ valor de $Z_{M K}$ para a série de precipitação anual (regional) do Cerrado foi $-1,5(<1,96)$ indicando que não houve tendência significativa a $95 \%$ de probabilidade. Entretanto, apesar de ser estatisticamente estacionária, graficamente há uma tendência geral de queda, conforme indica a Fig. 3.

Avaliando as estações separadamente, os resultados do teste de MK indicam que a maioria das estações estudadas não apresentou tendências significativas de queda ou aumento na precipitação anual, embora $71 \%$ dos postos analisados apresentassem tendências de redução da precipitação no período (não significativo). Esses resultados são consistentes com os reportados por Debortoli et al. (2015), que também não encontram tendências significativas na maioria das séries anuais de precipitação no bioma Cerrado.

É importante destacar que, dentre as 19 estações pluviométricas que apresentaram tendências significativas, 18 apresentaram tendências de redução da precipitação e uma apresentou tendência de aumento. A Fig. 4 apresenta espacialmente os resultados dos testes de MK e TS. Observa-se que a área central do Cerrado é caracterizada por estações com tendências de queda na precipitação, especialmente o estado de Goiás ( 9 postos), seguidos pelos 


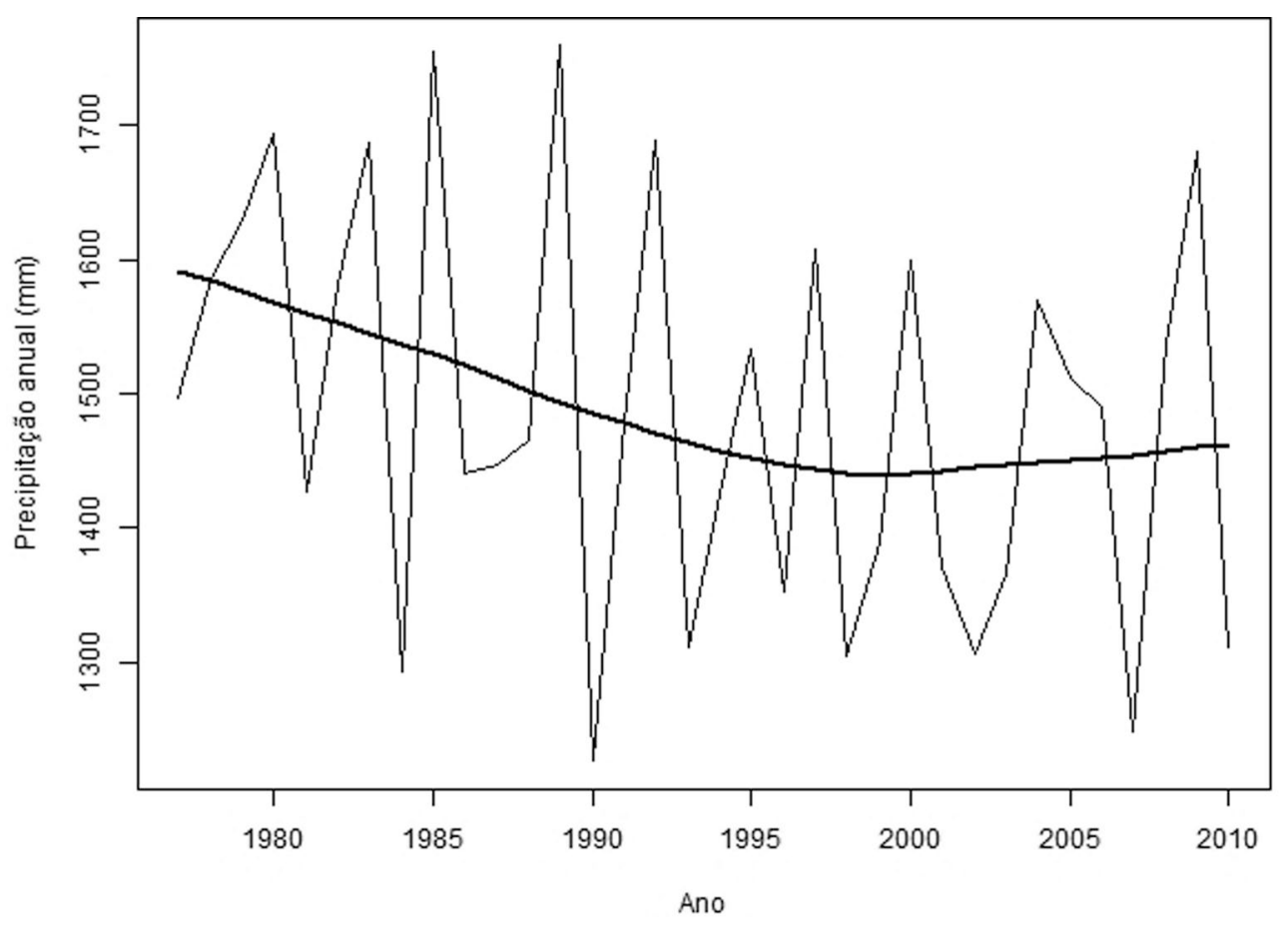

Figura 3 - Série histórica de precipitação anual $\left(\mathrm{mm}\right.$ ano $\left.^{-1}\right)$ do bioma Cerrado e a respectiva linha de tendência obtida pelo método do LOWESS.

estados do Tocantins (4 postos), Mato Grosso (3 postos), São Paulo (1 posto) e Mato Grosso do Sul (1 posto).

O Estado do Maranhão se diferencia dos demais pela predominância de estações com tendências de aumento de precipitação (embora não significativas), durante o período estudado. Dessas estações, cinco haviam sido investigadas por Silva et al. (2016), que encontraram resultados similares, com exceção da estação Chapadinha, a qual apresentou uma pequena tendência de queda na série em seu estudo. Esse aumento na precipitação anual no estado do Maranhão provavelmente está associado à influência do aumento da Temperatura da Superfície do Mar (TSM) no Oceano Atlântico nos últimos anos (Lee et al. 2011).

No período analisado, a única estação pluviométrica com tendência significativa de aumento da precipitação está localizada no extremo sul do bioma, em Itaberá - SP. Segundo Dufek e Ambrizzi (2008), o estado de São Paulo, incluindo a região desse posto pluviométrico, apresentou, entre 1950 e 1999, um aumento significativo da precipitação anual, o qual foi associado ao aumento do número de chuvas intensas no período.

A interpolação espacial dos resultados do teste de Theil-Sen dos 125 postos do Cerrado (Fig. 4) indica que houve uma redução média na precipitação de $3,7 \mathrm{~mm}$ por ano no bioma, ou seja, uma redução média de 125,8 mm durante os 34 anos avaliados. Essa redução corresponde a $8,4 \%$ da precipitação anual do bioma, com base na média pluviométrica anual obtida no presente estudo $(1.486 \mathrm{~mm})$.

Mais da metade do bioma exibiu uma ligeira queda na precipitação, com reduções entre $5,6 \mathrm{~mm} \cdot \mathrm{ano}^{-1}$ a $0 \mathrm{~mm}$. ano $^{-1}$ (Tabela 1). Reduções mais acentuadas foram registradas em $27 \%$ da área total do Cerrado, na porção central e oeste (Fig. 4). Por outro lado, o Maranhão, o sudeste Minas Gerais e o sul São Paulo, que correspondem a aproximadamente $17 \%$ da área total do bioma, apresentaram aumentos na precipitação no mesmo período. Os altos valores de desvio padrão observados nas classes do $\beta$ apresentados na Tabela 1 refletem a alta variabilidade da precipitação no bioma.

$\mathrm{O}$ teste de Pettitt indicou que 13 das 125 séries temporais individuais analisadas apresentaram rupturas significativas ao longo do período de estudo $(\alpha<0,05)$. Dentre essas, 12 tiveram tendências de redução da precipitação depois da ruptura, e somente uma apresentou aumento da precipitação após a quebra, corroborando com os resultados encontrados pelos testes de Mann Kendal e Theil-Sen. A série representando a média anual da precipitação no Cerrado não apresentou ruptura significativa.

As rupturas significativas nas séries ocorreram entre 1983 e 1998, sendo este último o ano com maior número de rupturas significativas observadas (3). Essas rupturas estão possivelmente associadas a fenômenos naturais ou a interferências nos registros dos dados pluviométricos (Cavalcanti et al., 2013). No entanto, como não foram encontradas inconsistências nos dados de precipitação, a primeira hipótese é a mais plausível.

Nesse sentido, foram observados eventos do fenômeno El-Niño nos anos de 1982/83, 1991/92, 1994/95, 1997/98, 2004/05, 2007 e 2010 (Cavalcanti et al. 2013; 


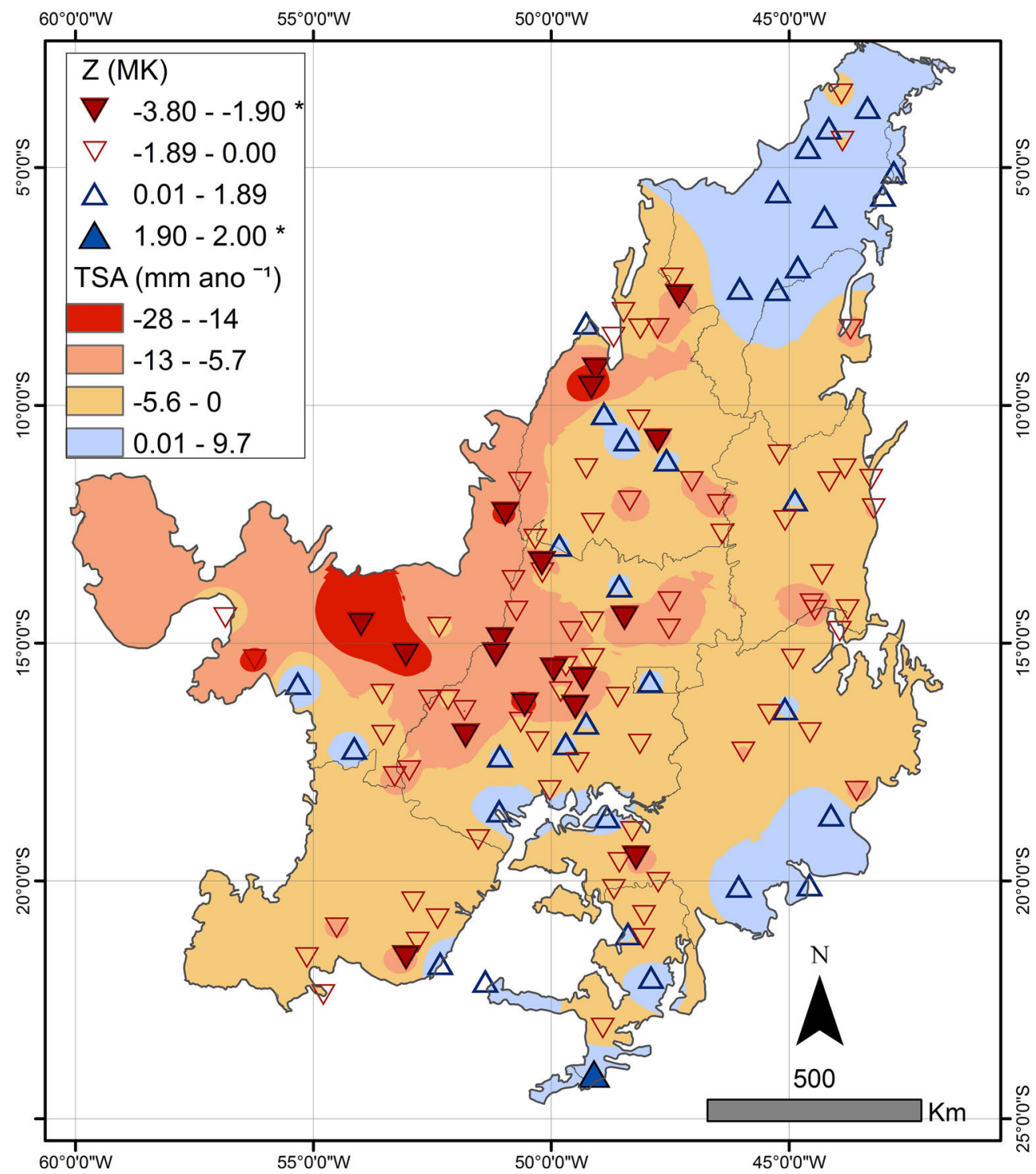

Figura 4 - Distribuição espacial das tendências de precipitação anual no Cerrado. TSA refere-se ao valor de $\beta$ (TS).

Tabela 1 - Variação da precipitação $(\beta)$ a partir da tendência das séries históricas anuais.

\begin{tabular}{lcccc}
\hline$\beta\left(\mathrm{mm}^{\left.-\mathrm{ano}^{-1}\right)}\right.$ & Média $\beta\left(\mathrm{mm} \cdot \mathrm{ano}^{-1}\right)$ & Desvio padrão $\beta\left(\mathrm{mm} \cdot \mathrm{ano}^{-1}\right)$ & Área $\left(\mathrm{km}^{2}\right)$ & 57.364 \\
\hline$-28 \mathrm{a}-14$ & $-18,38$ & 3,61 & 482.872 & 03 \\
$-13 \mathrm{a}-5,7$ & $-8,08$ & 1,73 & 1.143 .990 & 24 \\
$-5,6-0$ & $-3,22$ & 1,35 & 355.015 & 56 \\
$0,01-9,7$ & 3,26 & 2,09 & 2.039 .241 & 17 \\
Total & $-3,72$ & 4,74 & 100 \\
\hline
\end{tabular}

Oliveira et al. 2014). Sete séries pluviométricas apresentaram rupturas em anos de El Niño, sendo que em alguns desses anos (1983, 1994/95 e 1998) ocorreram outros eventos, como enfraquecimento da Zona de Convergência do Atlântico Sul (ZCAS) e anomalias negativas na TSM, que favorecem redução da precipitação no período (Debortoli et al. 2015; Lee et al. 2011).
Com relação as séries históricas mensais, dois meses se destacam dos demais em relação à quantidade de tendências estatisticamente significativas, de aumento ou redução da precipitação: janeiro e maio (Tabela 2). O mês de janeiro teve $75 \%$ dos postos com tendência de queda na precipitação, sendo que desse percentual, 18 estações $(14 \%)$ apresentaram resultados estatisticamente sig- 
Tabela 2 - Número de estações pluviométricas com tendência de precipitação na série histórica mensal: negativa estatisticamente significativa ( - $(*))$, negativa, sem significância $(-)$, nula $(0)$, positiva, sem significância $(+)$ e estatisticamente positiva $(+(*))$.

\begin{tabular}{lcccccccccccc}
\hline$Z_{\mathrm{MK}}$ & Jan & Fev & Mar & Abr & Mai & Jun & Jul & Ago & Set & Out & Nov & Dez \\
\hline$-\left(^{*}\right)$ & 18 & 2 & 6 & 8 & 18 & 9 & 10 & 12 & 11 & 8 & 6 & 5 \\
- & 75 & 42 & 46 & 59 & 52 & 96 & 74 & 83 & 86 & 71 & 59 & 66 \\
0 & 2 & 8 & 9 & 4 & 3 & 6 & 6 & 4 & 9 & 7 & 5 & 7 \\
+ & 23 & 71 & 62 & 52 & 41 & 14 & 35 & 26 & 19 & 37 & 51 & 46 \\
$+(*)$ & 7 & 2 & 2 & 2 & 11 & 0 & 0 & 0 & 0 & 2 & 4 & 1 \\
\hline
\end{tabular}

nificativos. Essa redução em janeiro também foi observada na série histórica do Cerrado, que exibiu $Z_{\mathrm{MK}}$ igual a $-1,2$ (não significativo) e uma redução na precipitação total de $68,1 \mathrm{~mm}$, equivalente a $26 \%$ da precipitação média de janeiro, pelo teste de TS (Tabela 3 ).

Os meses de estação seca (junho, julho, agosto e setembro) apresentaram tendências de redução na precipitação em $76 \%$ das 125 séries históricas analisadas. Para a série histórica regional do Cerrado, referente a junho, apresentou uma redução de $61 \%$ na precipitação durante os 34 anos estudados (Tabela 3). Ainda regionalmente, os meses de maio, setembro e outubro, que são considerados meses de transição entre a estação seca e a chuvosa no Cerrado, também indicaram uma queda considerável na precipitação, de $11 \%, 36 \%$ e $22 \%$, respectivamente. Esses resultados sugerem que está havendo uma intensificação da estação seca no Cerrado através da redução da intensidade da chuva e prolongamento dos meses secos, no caso da queda na precipitação nos meses de transição, tendências essas evidenciadas por outros autores (Costa e Pires, 2010; Debortoli et al. 2015).

Por outro lado, nos meses chuvosos, a queda isolada observada em janeiro indica que pode estar havendo um aumento dos períodos de veranicos, comuns no Cerrado (Assad et al. 1993). O aumento da sua frequência poderá, por sua vez, comprometer as lavouras de sequeiro, especialmente no pico da estação de crescimento (Assad et al. 1993; Hoffmann e Jackson, 2000). Uma possível explicação para essa redução seria um enfraquecimento da ZCAS durante esse mês, seja na frequência de ocorrência ou na capacidade de produzir precipitação (Chou et al. 2014).

Tendências de redução da precipitação nos meses chuvosos também foram observados por Almeida et al. (2017) para o estado do Tocantins, os quais atribuíram a diminuição observada como consequência dos eventos de El Niño-Oscilação Sul (ENOS), que ocorreram no início e fim da série temporal. Todavia, é improvável que o padrão observado no mês de janeiro, neste estudo, tenha sido influenciado por ENOS, pois os altos e baixos valores verificados na série temporal não coincidem com fenômenos de La Niña e El Niño, respectivamente (Boers et al. 2015; Almeida et al. 2017) (Fig. 5).

No conjunto, os testes de tendência aplicados às séries temporais de precipitação no Cerrado (MK, TS, LOWESS) mostraram resultados consistentes entre si, sugerindo uma tendência geral de redução na precipitação ao longo do período, considerando-se o bioma como um todo. Esse resultado é relevante já que a relativamente longa duração da série (34 anos) tenderia a neutralizar eventuais variabilidades interanuais, tendendo a manter a série temporal estacionária.

Dentre as possíveis causas para a tendência de redução observada está a influência das anomalias de TSM, tanto do oceano Atlântico quanto do Pacífico, as quais alteram os padrões do vento reduzindo o transporte de umidade para o continente e, assim, dificultando a formação de nuvens (PBMC, 2014). De acordo com Lee et al. (2011), uma parcela das reduções na precipitação ocorridas na região do Cerrado nas últimas décadas podem ser atribuídas à TSM. É esperado que o aumento da temperatura do oceano causado pelo aquecimento global nas próximas décadas intensifique essas anomalias e, consequentemente, as reduções da precipitação no bioma (PBMC, 2014).

Todavia, de acordo com Hoffmann e Jackson (2000), Lee et al. (2011) e Salazar et al. (2016), as reduções na precipitação do bioma a partir da década de 1970 eram esperadas como consequência do desmatamento em larga escala do Cerrado. Os mecanismos da relação entre o desmatamento regional/local e a redução da precipitação estão associados às mudanças nos processos biofísicos da superfície terrestre (albedo, rugosidade aerodinâmica e evapotranspiração) após a retirada da vegetação nativa. Em contrapartida, há redução da taxa de evapotranspi-

Tabela 3 - Tendências das séries históricas de precipitação média mensal do Cerrado (período 1977-2010).

\begin{tabular}{lcccccccccccc}
\hline Tendência & Jan & Fev & Mar & Abr & Mai & Jun & Jul & Ago & Set & Out & Nov & Dez \\
\hline$Z_{\mathrm{MK}}$ & $-1,2$ & 0,1 & 0,0 & $-0,2$ & $-0,7$ & $-1,3$ & 0,1 & $-0,8$ & $-1,1$ & $-1,2$ & $-0,3$ & $-0,7$ \\
$\beta(\mathrm{mm})$ & $-68,1$ & 6,8 & 2,4 & $-3,4$ & $-5,1$ & $-9,5$ & 0,5 & $-2,5$ & $-17,1$ & $-24,8$ & $-5,6$ & $-13,9$ \\
$\beta(\%)$ & -26 & 3 & 1 & -3 & -11 & -61 & 5 & -19 & -36 & -22 & -3 & -6 \\
\hline
\end{tabular}




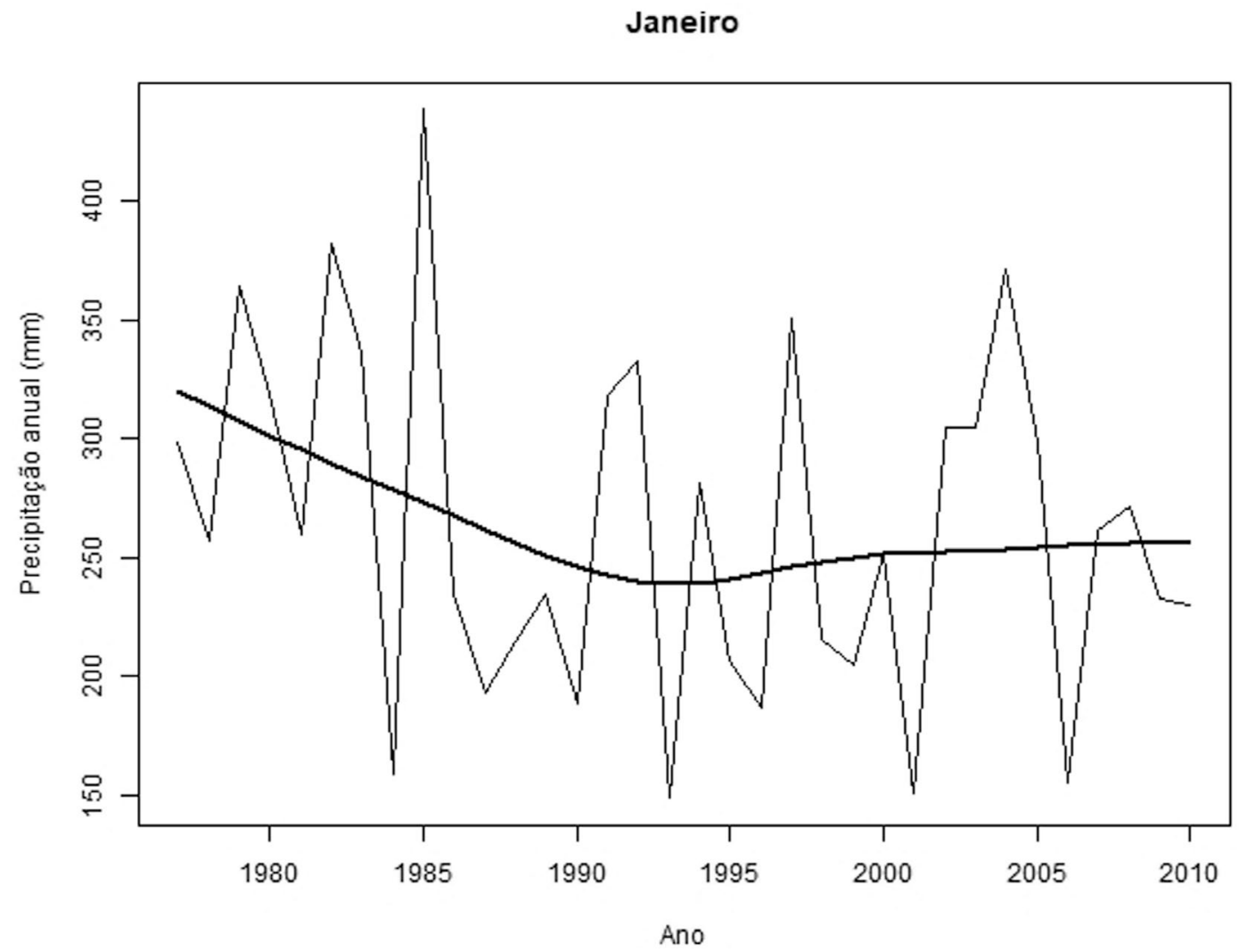

Figura 5 - Série histórica de precipitação $(\mathrm{mm})$ mensal do mês de janeiro do bioma Cerrado, obtida pelas médias mensais das 125 estações pluviométricas estudadas.

ração e dos processos convectivos, responsáveis pela formação de chuvas (Davin e De Noblet-Ducoudré, 2010).

\section{Conclusão}

Este estudo apresenta um quadro do comportamento da precipitação no bioma Cerrado nas últimas décadas, o que é fundamental para medidas de conservação da biodiversidade e de gestão dos recursos hídricos e do agronegócio do país. Das 125 estações pluviométricas estudadas no bioma Cerrado, 89 apresentaram tendências de redução na precipitação média anual, sendo que 18 estações mostraram queda estatisticamente significativa. Somente uma estação apresentou tendência significativa de aumento.

A amplitude da alteração na precipitação no Cerrado variou entre - $28 \mathrm{~mm} / \mathrm{ano}^{-1} \mathrm{a}+9,7 \mathrm{~mm} / \mathrm{ano}^{-1}$, sendo a média para todo bioma igual a $-3,7 \mathrm{~mm} / \mathrm{ano}^{-1}$, equivalente a uma redução de $8,4 \%$ na precipitação média no Cerrado $(125,8 \mathrm{~mm})$ durante os 34 anos analisados. A distribuição espacial das tendências nas séries de precipitação indica que as reduções mais importantes ocorreram nas porções central e oeste do bioma. Essas observações são consistentes com outros estudos, indicando que o bioma vem experimentando uma variabilidade climática importante nos últimos anos. Concomitante a mudança climática glo- bal, a possibilidade de efeitos do desmatamento sobre a precipitação do Cerrado parece ser relevante, uma vez que mais da metade da vegetação nativa do bioma foi convertida em outros usos.

\section{Referências}

ADLER, R.F.; HUFFMAN, G.J.; CHANG, A.; FERRARO, R.; XIE, P.P.; et al. The version-2 global precipitation climatology project (GPCP) monthly precipitation analysis (1979present). Journal of Hydrometeorology, v. 4, n. 6, p. 1147-1167, 2003.

AHMAD, I.; TANG, D.; WANG, T.F.; WANG, M.; WAGAN, B. Precipitation trends over time using Mann-Kendall and Spearman's rho Tests in Swat River Basin, Pakistan. Advances in Meteorology (on-line), v. 2015, 2015. <https://doi.org/10.1155/2015/431860>

ALIZADEH-CHOOBARI, O; NAJAFI, M.S. Extreme weather events in Iran under a changing climate. Climate Dynamics, v. 50, n. 1-2, p. 249-260, 2018.

ALMEIDA, C.T.; OLIVEIRA-JÚNIOR, J.F.; DELGADO, R.C.; CUBO, P.; RAMOS, M.C. Spatiotemporal rainfall and temperature trends throughout the Brazilian Legal Amazon, 1973-2013. International Journal of Climatology, v. 37, n. 4, p. 2013-2026, 2017. 
ALMEIDA, J.M.V. Índices de monitoramento e detecção de mudanças climáticas na região centro-oeste do Brasil. Dissertação. Mestrado em (Meteorologia), Departamento de Ciências Atmosféricas, Universidade Federal de Campina Grande. Brasília - DF, 2012. 236 f.

AGÊNCIA NACIONAL DE ÁGUAS (ANA); SUPERINTENDÊNCIA DE GESTÃO DA REDE HIDROMETEOROLÓGICA (SGH). Diretrizes e análises recomendadas para a consistência de dados pluviométricos. Agência Nacional Das Águas, Brasília - DF, 2011. 18 p.

ARANTES, A.E.; FERREIRA, L.G.; COE, M.T. The seasonal carbon and water balances of the Cerrado environment of Brazil: Past, present, and future influences of land cover and land use. ISPRS Journal of Photogrammetry and Remote Sensing, v. 117, p. 66-78, 2016.< https://doi.org/ 10.1016/j.isprsjprs.2016.02.008>

ASSAD, E.D.; SANO, E.E.; MASUTOMO, R.; DE CASTRO, L.H.R.; DA SILVA, F.A.M. Veranicos na região dos cerrados brasileiros: frequência e probabilidade de ocorrência. Pesquisa Agropecuária Brasileira, v. 28, n. 9, p. 9931003, 1993.

ÁVILA, A.; JUSTINO, F.; WILSON, A.; BROMWICH, D.; AMORIM, M. Recent precipitation trends, flash floods and landslides in southern Brazil. Environmental Research Letters, v. 11, n. 11, p. 114029, 2016.

BISHT, D.S.; CHATTERJEE, C.; RAGHUWANSHI, N.S.; SRIDHAR, V. Spatio-temporal trends of rainfall across Indian river basins. Theoretical and Applied Climatology, v. 132, n. 1-2, p. 419-436, 2018.

BOERS, N.; DONNER, R.V.; BOOKHAGEN, B.; KURTHS, J. Complex network analysis helps to identify impacts of the El Niño Southern Oscillation on moisture divergence in South America. Climate Dynamics, v. 45, n 3-4, p 619631, 2015.

CAVALCANTI, I.F.A; SILVEIRA V.P.; ALVES L.M. Características atmosféricas e oceânicas em anos de seca. In: Secas na Amazônia: causas e consequências. BORMA, S.; NOBRE, C. (orgs.) São Paulo: Oficina de Textos, p. 54-77, 2013.

CHOU, S.C.; LYRA, A.; MOURÃO, C.; DERECZYNSKI, C.; PILOTTO, I.; et al. Assessment of Climate Change over South America under RCP 4.5 and 8.5 Downscaling Scenarios. American Journal of Climate Change, v. 3, n. 5, p. 512-525, 2014.

COSTA, M.H.; PIRES, G.F. Effects of Amazon and Central Brazil deforestation scenarios on the duration of the dry season in the arc of deforestation. International Journal of Climatology, v. 30, n. 13, p.1970-1970, 2010.

DA SILVA, F.A.M.; ASSAD, E.D.; STEINKE, E.T.; MÜLLER, A.G. Clima do bioma Cerrado. In: Agricultura tropical: quatro décadas de inovações tecnológicas, institucionais e políticas. ALBUQUERQUE, A.C.S.; SILVA, A.G. (orgs.). Embrapa, Brasília, DF, p. 93-148, 2008.

DAVIN, E.L.; DE NOBLET-DUCOUDRÉ, N. Climatic Impact of Global-Scale Deforestation: Radiative versus Nonradiative Processes. American Meteorological Society, v. 23, n. 1, p. 97-111, 2010.

DE OLIVEIRA, L.F.; FIOREZE, A.P.; MEDEIROS, A.M.; SILVA, M.A. Comparação de metodologias de preenchimento de falhas de séries históricas de precipitação plu- vial anual. Revista Brasileira de Engenharia Agrícola e Ambiental-Agriambi, v. 14, n. 11, p.1186-1192, 2010.

DEBORTOLI, N.S.; DUBREUIL, V.; FUNATSU, B.; DELAHAYE, F.; OLIVEIRA, C.H.; RODRIGUES-FILHO, S.; SAITO, C.H.; FETTER, R. Rainfall patterns in the Southern Amazon: a chronological perspective (1971-2010). Climatic Change, v. 132, n. 2, p. 251-264, 2015.

DEBORTOLI, N.S.; DUBREUIL, V.; HIROTA, M.; RODRIGUES-FILHO, S.; LINDOSO, D.P.; NABUCET, J. Detecting deforestation impacts in Southern Amazonia rainfall using rain gauges. International Journal of Climatology. v. 37, n. 6, p. 2889-2900, 2016.

DUFEK, A.S.; AMBRIZZI, T. Precipitation variability in São Paulo State, Brazil. Theoretical and Applied Climatology, v. 93, n. 3-4, p. 167-178, 2008.

GOCIC, M., TRAJKOVIC, S., Analysis of changes in meteorological variables using Mann-Kendall and Sen's slope estimator statistical tests in Serbia. Global Planetary Change. v.100, p.172-182, 2013.< https://doi.org/10.1016/ j.gloplacha.2012.10.014>

HOFFMANN, W.A.; JACKSON, R.B. Vegetation- Climate feedbacks in the Conservation of Tropical Savanna to Grassland. American Metereological Society, v. 13, n. 9, p. 1593-1602, 2000.

INSTITUTO BRASILEIRO DE GEOGRAFIA E ESTATÍSTICA (IBGE). Produção agrícola municipal: Culturas temporárias e permanentes. Rio de Janeiro, RJ, v. 43, p. 1-62, 2016.

INTERGOVERNMENTAL PANEL ON CLIMATE CHANGE (IPCC). Managing the risks of extreme events and disasters to advance climate change adaptation: special report of the intergovernmental panel on climate change. FIELD, C.B.; BARROS, V.; STOCKER, T.F.; DAHE, Q. (orgs.). Cambridge University Press, Cambridge, UK, and New York, NK, USA. 582 p. 2012.

INTERGOVERNMENTAL PANEL ON CLIMATE CHANGE (IPCC). Climate Change 2014: Synthesis Report. Contribution of Working Groups I, II and III to the Fifth Assessment Report of the Intergovernmental Panel on Climate Change. PACHAURI, R.K.; MEYER, L.A. (orgs). IPCC, Geneva, Switzerland, 151 p., 2014.

INTERGOVERNMENTAL PANEL ON CLIMATE CHANGE (IPCC). Global warming of $1.5^{\circ} \mathrm{C}$. an IPCC Special Report on the Impacts of Global Warming of $1.5^{\circ} \mathrm{C}$ above preindustrial levels and related global Greenhouse Gas Emission pathways, in the context of strengthening the global response to the threat of climate change, sustainable development, and efforts to eradicate poverty. MASSON-DELMOTTE, V.P.; ZHAI, H.O.; PÖRTNER, D.; ROBERTS, J.; SKEA, P.R.; et al. (Orgs). Cambridge: Cambridge University Press. 562 p., 2018.

KENDALL, M.G. Rank Correlation Methods. Charles Griffin, London, 1975.

KUNDZEWICZ, Z.W.; ROBSON, A. Detecting trend and Other Changes in Hydrological Data. World Meteorological Organization Climate Programme-Water, World Climate Programme Date and Monitoring, WCDMP, WMO/TD, v. 45, n. 1013,158 p. 2000.

LEE, J.E.; LINTNER, B.R.; BOYCE, C.K.; LAWRENCE, P.J. Land use change exacerbates tropical South American 
drought by sea surface temperature variability. Geophysical Research Letter, v. 38, n. 19, p. 1-6, 2011.

LIMA, J.E.F.W. Situação e perspectivas sobre as águas do Cerrado. Ciência e Cultura, v. 63, n. 3, p. 27-29, 2011.

MALESKI, J.J.; MARTINEZ, C.J. Historical trends in precipitation, temperature and drought in the Alabama-Coosa-Tallapoosa and Apalachicola-Chattahoochee-Flint river basins. International Journal of Climatology, v. 37, n. 2, p. 583$595,2017$.

MANN, H.B. Non-parametric test against trend. Econometrica, v. 13, p. 245-259, 1945.

BRASIL; Ministério do Meio Ambiente (MMA). PPCerrado Plano de Ação para prevenção e controle do desmatamento e das queimadas no Cerrado: $2^{\mathrm{a}}$ fase (2014-2015). Ministério do Meio Ambiente, Brasília: DF, 2014. 132 p.

BRASIL; Ministério do Meio Ambiente (MMA). Plano de Ação para prevenção e controle do desmatamento e das queimadas: cerrado. Ministério do Meio Ambiente, Brasília, DF, 2011.

MOURATO, S.; MOREIRA, M.; CORTE-REAL, J. Interannual variability of precipitation distribution patterns in Southern Portugal. International Journal of Climatology, v. 30, n. 12, p. 1784-1794, 2010.

O'GORMAN, P.A. Precipitation extremes under climate change. Current climate change reports, v. 1, n. 2, p. 49-59, 2015.

OBREGÓON, G.; MARENGO, J. Variabilidade e tendências climáticas. In: Riscos das Mudanças Climáticas no Brasil: análise conjunto Brasil - Reino Unido sobre os impactos das mudanças climáticas e do desmatamento da Amazônia. MARENGO, J.A.; BETTS, R.; NOBRE, C.A.; SAMPAIO, G.; KAY, G.; et al. INPE, 2011. 56p.

OLIVEIRA, P.T.S.; NEARING, M.A.; MORAN, M.S.; GOODRICH, D.C.; WENDLAND, E.; et al. Trends in Water Balance components across the Brazilian Cerrado. Water Resources Research, v. 50, n. 9, p. 7100-7114, 2014.

Painel Brasileiro de Mudanças Climáticas (PBMC). Base científica das mudanças climáticas. Contribuição do: Grupo de Trabalho 1 do- Primeiro Relatório da Avaliação Nacional sobre Mudanças Climáticas. AMBRIZZI, T.; ARAÚJO, M. (Orgs.). COPPE, Universidade Federal do Rio de Janeiro, RJ, Brasil, 464 p. 2014.

PENEREIRO, J.C.; BADINGER, A.; MACCHERI, N.A.; MESCHIATTI, M. Distributions of Seasonal Average Temperature and Precipitation Trends in Brazilian Biomes. Revista Brasileira de Meteorologia, v. 33, n. 1, p. 97-113, 2018.

PETTITT, A.N. A non-parametric approach to the change point problem. Journal of the Royal Statistical Society Series C, Applied Statistics, v. 28, n. 2, p. 126-135, 1979.

POHLERT, T. (2018). Trend: Non-Parametric Trend Tests and Change-Point Detection. $\mathrm{R}$ package version 1.1.0. https://CRAN.R-project.org/package=trend.

RÍO, S.D.; HERRERO, L.; FRAILE, R.; PENAS, A. Spatial distribution of recent rainfall trends in Spain (1961-2006). International Journal of Climatology, v. 31, n. 5, p. 656667, 2011.

SÁ, E.A.S.; MOURA, C.N.D.; PADILHA. V.L; CAMPOS, C.G.C. Trends in daily precipitation in highlands region of
Santa Catarina, southern Brazil. Revista Ambiente \& Água, v. 13, n. 1, 2018.

SALAZAR, A.; BALDI, G.; HIROTA, M.; SYKTUS, J.; MCALPINE, C. Land use and land cover change impacts on the regional climate of non-Amazonian South America: A review. Global and Planetary Change, v. 128, p. 103119, 2015. <https://doi.org/10.1016/j.glopla cha.2015.02.009>

SALAZAR, A.; KATZFEY, J.; THATCHER, M.; SYKTUS, J.; WONG, K.; et al. Deforestation changes land-atmosphere interactions across South American biomes. Global and Planetary Change, v. 139, p. 97-108, 2016.

SALVIANO, M.F.; GROPPO, J.D.; PELLEGRINO, G.Q. Trends Analysis of Precipitation and Temperature Data in Brazil. Revista Brasileira de Meteorologia, v. 31, n. 1, p. 64-73, 2016.

SANOGO, S.; FINK, A.H.; OMOTOSHO, J.A.; BA, A.; REDL, R.; et al. Spatial - temporal characteristics of the recent rainfall recovery in West Africa. International Journal of Climatology, v. 35, n. 15, p. 4589-4605, 2015.

SAYEMUZZAMAN, M.; JHA, M.K. Seasonal and annual precipitation time series trend analysis in North Carolina, United States. Atmospheric Research, v. 137, p. 183-194, 2014. <http://dx.doi.org/10.1016/j.atmosres.2013.10.012 >

SEN, P.K. Estimates of the regression coefficients based on Kendall's tau. Journal of American Statistical Association, Alexandria, v. 63, n. 324, p. 1379-1389, 1968.

SHARMA, S.; SAHA, A.K. Statistical analysis of rainfall trends over Damodar River basin, India. Arabian Journal of Geoscience, v.10, n. 15, p. 319, 2017.

SHUMWAY, R.H; STOFFER, D.S. Time Series Analysis and its application with R examples. Ed.3. New York: Springer Science, p. 1-102, 2011.

SILVA, F.B.; SANTOS, J.R.N.; FEITOSA, F.E.C.S.; SILVA, I.D.C.; ARAÚJO, M.L.S.; et al. Evidências de Mudanças Climáticas na região de transição da Amazônia-Cerrado no estado do Maranhão. Revista Brasileira de Meteorologia, v. 31, n. 13, p. 330-336. 2016.

SPERA, S.A.; GALFORD, G.L.; COE, M.T.; MACEDO, M.N.; MUSTARD, J.F. Land-use change affects water recycling in Brazil's last agricultural frontier. Global Change Biology, v. 22, n. 10, p. 3405-3413, 2016.

STRASSBURG, B.B.N.; BROOKS, T.; FELTRAN-BARBIERI, R.; IRIBARREM, A.; CROUZEILLES, R.; et al. Moment of truth for the Cerrado hotspot. Nature Ecology \& Evolution, v. 1, n. 4, p. 1-3, 2017.

TAXAK, A.K.; MURUMKAR, A.R.; ARYA, D.S. Long term spatial and temporal rainfall trends and homogeneity analysis in Wainganga basin, Central India. Weather and Climate Extremes, v. 4, p. 50-61, 2014. <https://doi.org/ 10.1016/j.wace.2014.04.005 >

THEIL, H. A rank-invariant method of linear and polynomial analysis, Part 3. Proceedings of Koninalijke Nederlandse Akademie van Weinenschatpen A, v. 53, p. 1397-1412, 1950.

TUCCI, C.E.M. Hidrologia: ciência de aplicação. Editora da UFRGS/ABRH, Porto Alegre, RS. 943p. 2012. 
VILANOVA, M.R.N. Tendências hidrológicas na região do alto rio Mogi-Guaçu, sul de Minas Gerais. Revista Agrogeoambiental, v. 6, n. 3, 2014.

WORLD METEOROLOGICAL ORGANIZATION (WMO). Calculation of monthly and annual 30-year standard normals: WMO-TD/No. 341. World Meteorological Organization, Geneva, 1989.

ZANDONADI, L.; ACQUAOTTA, F.; FRATIANNI, S.; ZAVATTINI, J.A. Changes in precipitations extremes in Brazil (Paraná River Basin). Theoretical and Applied Climatology, v. 123, n. 3-4, p. 741-756, 2016.

\section{Endereços de Internet}

AGÊNCIAS NACIONAL DAS ÁGUAS (ANA) - HIDROWEB Disponível em: http://www.snirh.gov.br/hidroweb/apresen tacao.

License information: This is an open-access article distributed under the terms of the Creative Commons Attribution License (type CC-BY), which permits unrestricted use, distribution and reproduction in any medium, provided the original article is properly cited. 